\title{
On non-stationarity of ENSO
}

\author{
Andrew R. Solow and Amit Huppert \\ Woods Hole Oceanographic Institution, Woods Hole, Massachusetts, USA
}

Received 22 July 2003; revised 22 July 2003; accepted 15 August 2003; published 13 September 2003.

[1] Evolutionary spectral analysis has been used to study changes through time in the variability of ENSO-related time series. However, the significance of estimated evolutionary spectra has not been formally assessed. This paper describes a test for non-stationarity based on an estimate of the evolutionary spectrum and a time series bootstrap procedure. The test is applied to the seasonal time series of sea level pressure at Darwin. No significant nonstationarity is found. INDEX TERMS: 1620 Global Change: Climate dynamics (3309); 1635 Global Change: Oceans (4203); 4215 Oceanography: General: Climate and interannual variability (3309); 4522 Oceanography: Physical: El Nino. Citation: Solow, A. R., and A. Huppert, On non-stationarity of ENSO, Geophys. Res. Lett., 30(17), 1910, doi:10.1029/2003GL018225, 2003.

\section{Introduction}

[2] El Niño-Southern Oscillation (ENSO) is the dominant source of interannual variability in global climate. There is considerable interest in understanding the long-term historical stability of ENSO variability [e.g., Trenberth and Hoar, 1996, 1997; Harrison and Larkin, 1997; Rajagopalan et al., 1997]. This interest stems in part from a possible connection between ENSO variability and large-scale warming. A natural way to detect changes over time in the variability of a time series of a direct or indirect measure of ENSO is through evolutionary spectral analysis [Priestley, 1981]. As described in more detail below, this involves estimating the spectral density function locally in time. Although evolutionary spectra have been estimated for ENSO-related time series by Mann et al. [2000], Urban et al. [2000], and others, no formal assessment of the significance of these estimates appears to have been made. This paper describes a test for non-stationarity based on an estimate of the evolutionary spectrum. Significance is assessed through a time series bootstrap procedure. The test is applied to a seasonal time series of sea level pressure at Darwin and no evidence of non-stationarity is found.

\section{Method}

[3] Consider a time series $X=\left(X_{1}, X_{2}, \ldots, X_{n}\right)$ of length $n$. Interest centers on testing the null hypothesis $H_{o}$ that the spectral density function of $X$ is stationary over time against the alternative hypothesis $H_{1}$ that the spectral density function is non-stationary. Let $X_{t}(m)=\left(X_{t-\mathrm{m}}, X_{t-m+1}, \ldots\right.$, $\left.X_{t+m}\right)$ be the subseries of $X$ of length $2 m+1(m<(n-1) /$ 2) centered at time $t(t=m+1, m+2, \ldots, n-m)$ and let:

$$
I_{t}\left(\omega_{j} ; m\right)=\frac{1}{2 m+1}\left|\sum_{s=t-m}^{t+m} X_{s} \exp \left(-i \omega_{j} s\right)\right|^{2}
$$

be the periodogram of the subseries $X_{t}(m)$, where $\omega_{j}=2 \pi j$ l $(2 m+1), j=1,2, \ldots, m$. We will refer to $I_{t}\left(\omega_{j} ; m\right)$ with $t$ fixed as the local periodogram and we will refer to the ordered series of local periodograms $I_{m+1}\left(\omega_{j} ; m\right), I_{m+2}\left(\omega_{j} ; m\right), \ldots$, $I_{n-m}\left(\omega_{j} ; m\right)$ as the evolutionary periodogram.

[4] Under $H_{o}, I_{t}\left(\omega_{j} ; m\right)$ has an approximately exponential distribution with mean $f\left(\omega_{j}\right)$, where $f(\omega)$ is the true spectral density function of $X$ [Priestley, 1981]. It follows that, under $H_{o}, f\left(\omega_{j}\right)$ can estimated by the average over time of the local periodograms:

$$
\hat{f}\left(\omega_{j} ; m\right)=\frac{1}{n-2 m} \sum_{t=m+1}^{n-m} I_{t}\left(\omega_{j} ; m\right)
$$

A test of the goodness of fit of this estimate to the individual local periodograms can be based on the exponential deviance:

$$
D=-2 \sum_{t=m+1}^{n-m} \sum_{j=1}^{m} \log \left(\frac{I_{t}\left(\omega_{j} ; m\right)}{\hat{f}\left(\omega_{j} ; m\right)}\right)-\frac{I_{t}\left(\omega_{j} ; m\right)-\hat{f}\left(\omega_{j} ; m\right)}{\hat{f}\left(\omega_{j} ; m\right)}
$$

[McCullagh and Nelder, 1989]. To use $D$ to test for nonstationarity, it is necessary to know its distribution under $H_{o}$. When $\left|t-t^{\prime}\right|<2 m+1$, the subseries $X_{t}(m)$ and $X_{t^{\prime}}(m)$ overlap, $I_{t}\left(\omega_{j} ; m\right)$ and $I_{t^{\prime}}\left(\omega_{j} ; m\right)$ are not independent, and the standard distributional result for the deviance, which assumes independent observations, does not hold. Instead, the statistical significance of the observed value of $D$ can be assessed using the so-called sieve bootstrap [Bühlmann, 2002].

[5] The sieve bootstrap, which was specifically developed for time series data, proceeds by fitting a stationary autoregressive model of order $p$ to the complete time series $X$. This model has the form:

$$
X_{t}-\mu=\sum_{j=1}^{p} \phi_{j}\left(X_{t-j}-\mu\right)+\varepsilon_{t}
$$

For fixed $p$, the parameters $\mu$ and $\phi_{1}, \phi_{2}, \ldots, \phi_{p}$ can be estimated by the method of maximum likelihood (ML) and, by fitting models of different order, the estimated order $\hat{p}$ can be found by minimizing Akaike's Information Criterion (AIC). Details are available in most texts on time series analysis [e.g., Brockwell and Davis, 1991]. Let:

$$
R_{t}=\left(X_{t}-\hat{\mu}\right)-\sum_{j=1}^{\hat{p}} \hat{\phi}_{j}\left(X_{t-j}-\hat{\mu}\right) \quad t=p+1, p+2, \ldots, n
$$

be the residuals from the fitted model, where $\hat{\mu}$ and $\hat{\phi}_{1}$, $\hat{\phi}_{2}, \ldots, \hat{\phi}_{p}$ are the ML estimates of the corresponding parameters. Form a bootstrap time series $X^{*}$ according to:

$$
X_{t}^{*}=\hat{\mu}+\sum_{j=1}^{\hat{p}} \hat{\phi}_{j}\left(X_{t-j}^{*}-\hat{\mu}\right)+R_{t}^{*} \quad t=p+1, p+2, \ldots, n
$$




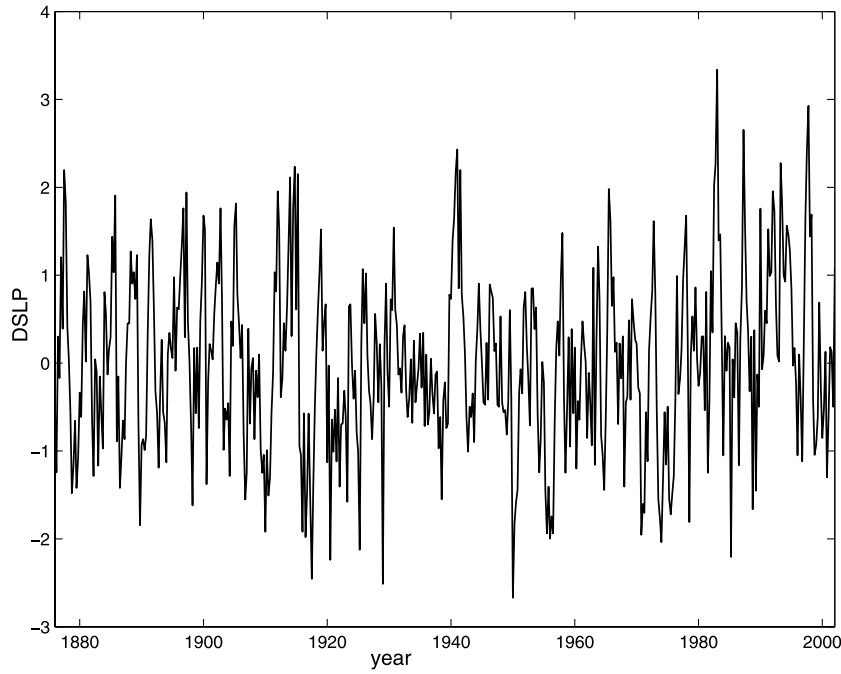

Figure 1. Standardized seasonal time series of sea level pressure at Darwin, 1876-2002.

where $X_{t}^{*}=X_{t}$ for $t=1,2, \ldots, \hat{p}$ and $R_{t}^{*}$ is sampled at random with replacement from the observed residuals. Treating the bootstrap series $X^{*}$ in exactly the same way as the original data, find the value $D^{*}$ of the deviance. Repeat the procedure a large number of times and estimate the significance level by the proportion of bootstrap series for which $D^{*}$ exceeds the observed deviance $D$.

\section{Results}

[6] This section presents the results of applying the method described in the previous section to test for nonstationarity in the standardized seasonal time series of sea level pressure at Darwin for the period 1876-2002. This time series, which is shown in Figure 1, has a total length of $n=508$ and is an updated version of the time series analyzed by Trenberth and Hoar [1996]. Figure 2 shows the evolutionary periodogram for this time series for $m=80$. For this choice of $m$, each subseries covers approximately

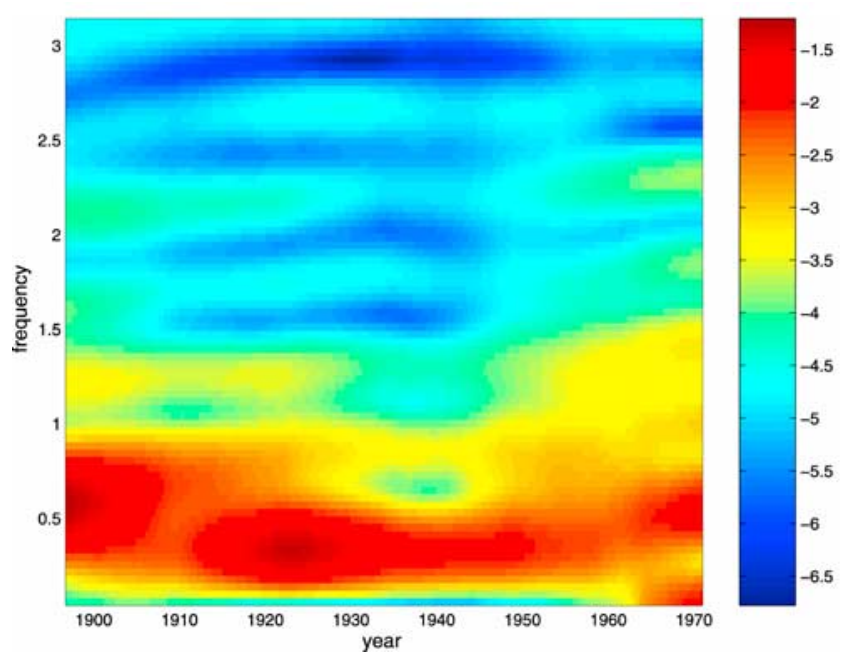

Figure 2. The log evolutionary periodogram for the time series is Figure 1 with $\mathrm{m}=80$.

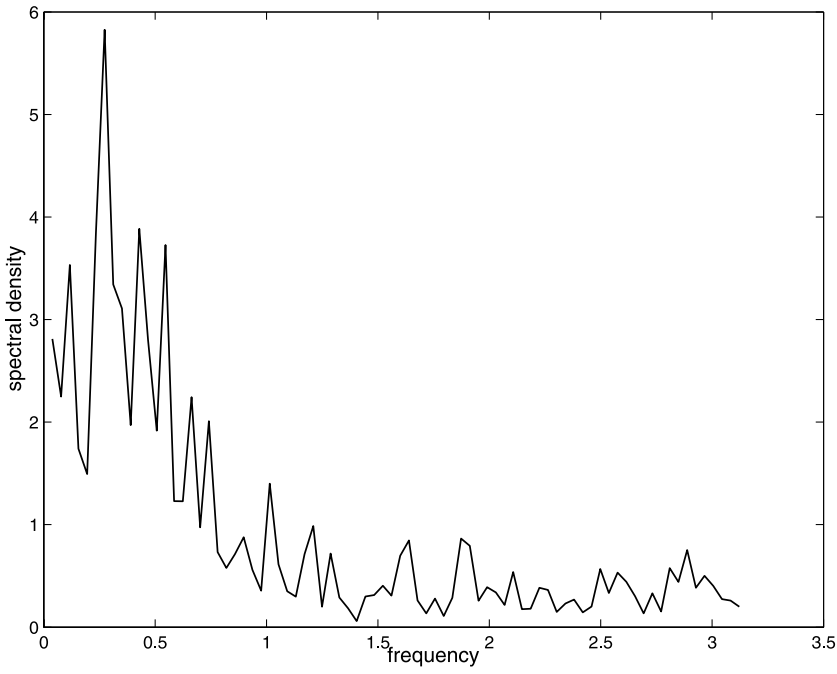

Figure 3. Spectral estimate based on the evolutionary periodogram in Figure 2.

40 years. In Figure 3, the spectral estimate $\hat{f}\left(\omega_{j}\right)$ is shown. The deviance for this estimate is $D=25142$.

[7] Turning to the sieve bootstrap, the order selected by the AIC is $\hat{p}=4$. The estimates of the autoregressive parameters for this model are $\hat{\phi}_{1}=0.51, \hat{\phi}_{2}=0.18, \hat{\phi}_{3}=-0.07$, and $\hat{\phi}_{4}=-0.08$. The initial standardization of the time series ensures that the mean $\mu$ is 0 . Of 100 bootstrap series generated from this fitted model, 60 had deviance values $D^{*}$ larger than 25142 , for an estimated significance level of 0.60 . We repeated the test using selected values of $m$ between 80 and 200 and fitted AR models of order 3 and 5 with no qualitative change in the estimated significance level. This analysis provides no evidence to support non-stationarity in the time series in Figure 1. Similar results were found for a time series if sea surface temperature in the so-called Niño-3 region.

\section{Discussion}

[8] The purpose of this paper has been to test for nonstationary variability in an ENSO-related time series. The test is based on a simple estimate of the evolutionary spectrum in conjunction with a time series bootstrap procedure. It is worth pointing out that, under the alternative hypothesis of non-stationarity, the evolutionary periodogram is not a good estimate of the evolutionary spectrum. However, interest here has centered on hypothesis-testing and not on estimation and the evolutionary periodogram is both convenient and appropriate for this purpose.

[9] The results of the previous section indicate that local variations in ENSO over the past century - at least as reflected in sea level pressure at Darwin and sea surface temperature in the Niño-3 region - are not inconsistent with overall stationarity. The test applied here considered variability at the full range of frequencies. The analysis could be sharpened by restricting attention to a range of frequencies over which non-stationarity is of particular interest. This range would have to be specified independently and, in particular, could not be chosen on the basis of the evolutionary periodogram (or other evolutionary spectral estimate). 
[10] Acknowledgments. The authors thank two anonymous reviewers for their comments.

\section{References}

Brockwell, P. J., and R. A. Davis, Time Series: Theory and Methods, 2nd ed., 527 pp., Springer-Verlag, New York, 1991.

Bühlmann, P., Bootstraps for time series, Stat. Sci., 17, 52-72, 2002.

Harrison, D. E., and N. K. Larkin, Darwin sea level pressure, 1876-1996:

Evidence for climate change?, Geophys. Res. Lett., 24, 1779-1782, 1997.

Mann, M. E., R. S. Bradley, and M. K. Hughes, Long-term variability in the El Niño/Southern Oscillation and associated teleconnections, in El Niño and the Southern Oscillation: Multiscale Variability and its Impacts on Natural Ecosystems and Society, edited by H. Diaz and V. Markgraf, pp. 321-372, Cambridge Univ. Press, New York, 2000.

McCullagh, P., and J. Nelder, Generalized Linear Models, Chapman and Hall, New York, 1989.
Priestley, M. B., Spectral Analysis and Time Series, 890 pp., Academic, San Diego, Calif., 1981.

Rajagopalan, B., U. Lall, and M. A. Cane, Anomalous ENSO occurrences: An alternative view, J. Clim., 10, 2351-2357, 1997.

Trenberth, K., and T. Hoar, The 1990-1995 El Niño-Southern Oscillation Event: Longest on record, Geophys. Res. Lett., 23, 57-60, 1996.

Trenberth, K., and T. Hoar, El Niño and climate change, Geophys. Res. Lett., 24, 3057-3060, 1997.

Urban, F. E., J. E. Cole, and J. T. Overpeck, Influence of mean climate change on climate variability from a 155 -year tropical Pacific coral record, Nature, 407, 989-993, 2000.

A. R. Solow and A. Huppert, Woods Hole Oceanographic Institution, Woods Hole, MA 02543, USA. (asolow@whoi.edu; ahuppert@whoi.edu) 